\title{
NOMBRAMIENTO DE \\ GASPAR DE PACHS, COMO \\ PRIMER MAESTRO RACIONAL \\ DEL REINO DE MALLORCA \\ (A. 1451)
}

Sara Cunchillos Plano (†)

Los fondos documentales del Archivo del Real Patrimonio de Cataluña, que fueron trasladados al Archivo de la Corona de Aragón en el año 1930, contienen pequeñas «series especiales» inventariadas precisamente por el doctor Udina en marzo de 1947; entre ellas, la que lleva como signatura Letra $C$ corresponde a la Real Procuración de Mallorca; y siguiendo su inventario, vemos que desde el año 1437 al 1444, es Procurador Real Lázaro de Loscos, el cual fue hasta 1427 lugarteniente de la Gobernación. En 1445 y 1446, es Procurador Gaspar de Pachs; desde 1446 al 1453 no figura nadie como Procurador, mientras que, en 1453 y 1454, lo es Albertí. Observamos, pues, una laguna de siete años (1446-1453) en los que parece ser que dicha Procuración Real de Mallorca no tiene «regent». Sin embargo, los documentos que sirven de soporte a nuestras consideraciones pertenecen a los años 1451 y 1452 y en ellos se habla del Procurador Albertí, por lo que cabría suponer cierta contradicción; pero eso es lo que reflejan los documentos.

La lectura meditada del trabajo que Álvaro Santamaría presentó al IV Congreso de Historia de la Corona de Aragón en 1955, publicado en 1976, nos dio una visión del confusionismo en que estuvo sumido el Reino en la primera mitad del siglo XV, proceso que desembocó en el levantamiento foráneo de los años 
1450-54. Disposiciones del rey desde Nápoles, disposiciones de la reina desde Barcelona, embajadas, desorientación, informes contradictorios que aumentaban la confusión; intrigas y dinero, las armas empleadas. Se disputaron la lugartenencia tres personalidades y entre bastidores, Juan Albertí, personaje que se fuga a Nápoles después de su arresto en Barcelona por un gran desfalco financiero. Don Alfonso, hábil político, consigue la convivencia pacífica entre las gentes de la Ciudad y las de las villas de Mallorca, al optar por el régimen de concordia y no por el de franqueza ni por el de pragmática.

Exponemos este panorama político porque el documento de confirmación en el cargo de maestro racional, a favor de Gaspar de Pachs, que transcribiremos, pudiera ser que no llegara a tener plena efectividad, ya que no hemos visto mencionado este cargo en cuantos estudios hemos leído sobre Instituciones en Mallorca. Sin embargo, la creación del oficio de maestro racional y su posterior confirmación fue un hecho; lo prueban los documentos que transcribiremos. El oficio se crea, se organiza y se le concede local digno y adecuado; los documentos en cuestión podrian acabar con la idea sostenida por algunos historiadores de que en Mallorca no existió nunca maestro racional, sino únicamente procurador, ya que dicen taxativamente Concessionis atque Comissionis et Creacionis Officii.

\section{COMENTARIO AL DOCUMENTO}

Con el fin de obviar las incomodidades de los desplazamientos a Barcelona para rendir cuentas al Maestro Racional de Cataluña, y «al igual que se ha hecho en los reinos de Aragón y Valencian, el rey cree que el varón más idóneo para desempeñar el cargo, "el nuevo cargo", de maestro racional en el reino de Mallorca es Gaspar de Pachs, Consejero y Conservador del Real Patrimonio en dicho Reino y cuya fidelidad y capacidad para el mismo ha demostrado. El Privilegio de creación y concesión de dicho oficio (que no existía en el reino de Mallorca) está otorgado por Alfonso el Magnánimo el 18 de enero de 1451, en Nápo- 
les, en Castello Novo y dice: «Constituímos de nueva creación y ordenamos el Maestro Racional de nuestra Curia en el reino de Mallorca, del mismo modo y forma que está instituído ya en Aragón y Valencia». Efectivamente, se toma como modelo el régimen de funcionamiento del de Valencia. Será cargo vitalicio y deberá ejercerlo con todos los honores, potestades, libertades e inmunidades que su similar de Valencia. Estará constituido por un ministro, que es el Maestro Racional, con 300 libras de salario; dos escribanos, el primero con 100 libras anuales y será el lugarteniente del Maestro Racional. El segundo escribano, con 75 libras; y completará la plantilla un verguer o portero, con 25 libras. El primer escribano es Juan Mateo y pasa a ser lugarteniente desde enero de 1451 aunque su nombramiento lleva fecha de 25 de octubre del mismo año. El escribano segundo es Jaime Landrich; y el verguer o portero es Bartolomé Zaragoza.

Este privilegio escrito por Arnaldo Fonolleda y visado por Pedro de Capdevila, tesorero en funciones de conservador, va dirigido a la reina María; en él especifica las obligaciones del Maestro Racional: Oir, examinar, discutir y ver, repasar todas las cuentas y raciones de los administradores, concluir, admitir y definir dichas cuentas, cosa que antes se hacía en la Curia Real, en el Principado de Cataluña, tanto del reino de Mallorca como de las Islas Adyacentes, Ibiza y Menorca.

El lugar asignado será el interior del Castillo Real de la Ciudad de Mallorca, considerado lugar suficiente y apto y además el más idóneo, por albergar en el mismo lugar el Archivo donde se guardan los libros, cuadernos, cautelas, ápocas y escrituras que han sido otorgadas.

Deberá observar las ordinaciones, estatutos y todas las Instituciones y tendrá la superioridad y prerrogativas que de antiguo tienen quienes detentan tal magistratura.

En otro documento el rey se dirige al Procurador Real y dice: «Como ya sabeis, hemos creado en el reino de Mallorca el Maestro Racional de nuestra Corte a favor de Gaspar de Pachs, con su salario, que le será pagado cada año y a su debido tiempo». Esta advertencia estará motivada por el préstamo de mil duca- 
dos que Gaspar de Pachs hizo al rey, de los cuales se le dieron letras de cambio dirigidas al Procurador.

Que Gaspar de Pachs llegara a ejercer el cargo es problemático, puesto que con fecha 25 de octubre de 1451 pactó una concordia de buena voluntad con Juan Albertí, para evitar los inconvenientes que pudieran derivarse de dicho nombramiento, al ser de más antigüedad el cargo de Procurador. El rey ordena que, en tanto no se extinga el Oficio por muerte de Juan Albertí, éste preceda en todo al Maestro Racional; pero cuando el Oficio de Procurador venga a manos de otra persona, por fallecimiento o por renuncia, suspensión u otra causa, pase el Maestro Racional a ocupar el lugar de más preeminencia como ocurre en el Maestro Racional «de otras tierras, como en el reino de Valencia».

Desde que en 1915 Félix Ferraz publicara su tesis doctoral, apenas se han dedicado estudios al tema del Maestro Racional. Así lo confirma la ponencia del doctor Font i Rius en el IV Congreso de Historia de la Corona de Aragón (Palma de Mallorca, 1955), publicada en 1976 (p. 214), en la que cita el trabajo de Ángeles Masiá, «Una pragmática de Juan II» (publicado en Hispania XXXVIII) y el del doctor Mateu Llopis «Los fondos documentales del Maestro Racional en el Archivo del Reino de Valencia»; y se dice en la citada ponencia «de esta reglamentación (Ordenanzas del Ceremonioso) se vivió fundamentalmente hasta los días del Rey Católico».

Efectivamente, poca bibliografia se conoce sobre el tema'. El trabajo de Ferraz nos informa ampliamente de la estructura y

1 Ferraz, Félix, El Maestre Racional y la Hacienda Foral valenciana.

MAteu y Llopis, Felipe, Los fondos documentales del Maestro Racional en el Archivo del Reino de Valencia.

Mateu Ibars, M. Dolores, Gaspar Gil Polo, primer coadjutor de Maestro Racional en Valencia.

Masix, Ángeles, «Una pragmática de Juan II», Hispania XXXVIII.

Cunchil.os, Sara, "Algunas vicisitudes del "Oficio" del Maestro Racional de Aragón, en Zaragoza (siglo xviı)"s, en X Congreso de Historia de la Corona de Aragón.

Montaciut, Tomás, Tesis doctoral sobre el Maestro Racional de Cataluña, leida en la Facultad de Derecho de Barcelona. Todavía no publicada. 
funciones del Oficio de Maestro Racional, Contador supremo del Reino, interventor en todos los oficios de la Hacienda, con una acertada organización de sus funcionarios y de su contabilidad. Sabemos que en un principio fue cargo único para toda la Corona de Aragón; pero Alfonso el Magnánimo en 1419 estableció la residencia fija del de Valencia en dicha ciudad, para oír y liquidar todas las cuentas, incluso las de los tesoreros de la reina y de todos los oficiales de la Curia Real y archivarlas en el Archivo del Real Palacio ${ }^{2}$.

Los privilegios y la jurisdicción del Oficio fueron en progresivo aumento, hasta converger en él los máximos honores; pero también sus deberes fueron muy estrictos y rigurosos, siempre siguiendo las citadas Ordenanzas del Ceremonioso del año 1344. Sus tres Libros Notaments Comuns, Albarans de Comptes y Llibre Ordinari, guardados bajo llave secretamente, por él o por su lugarteniente.

Si el privilegio de Alfonso el Magnánimo (Lérida, 1420) confirmó el establecimiento del Oficio en Valencia, suponemos que por las mismas fechas lo haría para el Reino de Aragón, con residencia en Zaragoza ${ }^{3}$ puesto que sabemos que allí funcionó a partir de 1417; pero no se ha localizado, que sepamos, el documento de creación del mismo. Respecto al de Mallorca, como se ha dicho, funcionaba un Procurador que personalmente iba a Barcelona a rendir cuentas al Maestro Racional de Cataluña, hasta que se confirma la creación del cargo para el Reino de Mallorca; podemos aportar varios documentos que transcribiremos, asi como la concordia de buena voluntad que Gaspar de Pachs pactó para no entorpecer la actuación del intrigante Albertí como Procurador. Bien patente queda en dicha concordia que, mientras viviera el anciano Procurador, Gaspar de Pachs quedaría relegado a un discreto segundo término, pero ostentando el cargo, «de cuyo nombramiento no puede quedar duda».

El hallazgo de los documentos que a continuación transcribi-

2 Fueros extravagantes, tomo II, fol. 40.

3 "Algunas vicisitudes del "Oficio del Maestro Racional" de Aragón, en Zaragoza», en $X$ Congreso de Historia de la Corona de Aragón. 
remos significa una contribución al estudio de esta Institución, tema todavía poco conocido.

\section{APÉNDICE}

Pro Gaspare de Pachs. CONFIRMACIO OFFICII MAGISTRI RACIONALIS MAIORICARUM.

Nos Alfonsus Dei gracia rex Aragonum, etc. Ordinavimus superioribus diebus mature deliberateque acque ex certa sciencia nostra dilectum consiliarium nostrum GASPAREM de PACHS, civem civitatis Maioricarum in MAGISTRUM RACIONALEM Curie nostre in dicto regno Maioricarum ut omnia hec clarissime patent serie privilegii concessionis acque comissionis et CREATIONIS OFFICII eiusdem tenoris sequentis. Nos Alfonsus Dei gracia rex Aragonum, etc. Optimi acque sagacissimi principis est ea que inutiliter et cum detrimento curie et subditorum fiunt licet a retroprincípibus ordinata sint in melius commutare cum non minori sed longe alciori cura ingenio, industria et rerum experiencia in corrigendo inventa quam in inveniendo opus sit verum cum in hodiernum usque diem usitatum sit quod procurator regius regni Maioricarum eo quod in eodem regno non est neque hactenus fuit MAGISTER RACIONALIS compota et raciones of ficialium et particularium dicti regni administratorum audiebat et diffiniebat et inde de sua administratione et dictorum officialium et administratorum predictorum coram MAGISTRO RACIONALI curie nostre in civitate Barchinone reddebat racionem quo ordine non omnia male et in preiudicium curie nostre acta ad noticiam dicti Magistri Racionalis veniebant preterea propter locorum distanciam multa compota presentium et preteritorum administratorum inaudita atque indifinita sunt in non parvam curie nostre jacturam. Cum nemo ut accepimus administratorum sit qui nobis non teneatur in maximis pecuniarum quantitatibus. Volentes iccirco huic tam evidenti curie nostre incomoditati obviare et utilitati nostre salubriter consulere, deliberavimus mature quidem et digeste ut in regnis Aragonum et Valencie actum est in reg. no Maioricarum agere hoc est virum aliquem peritum et de nobis benemeritum proprium et dícatum tamen ad rationes et compota administratorum omnium audiendum et quod per alios Magistros Racionales circa eorum officia fit faciendum illius regni Magistrum Racionalem ordinare; dumque hec animo voluebamus occurrit quod nemini magis idoneo de dicto officio providere possemus quam vobis GASPARI DE PACHS, CONSILIARIO ET CONSERVATORI nostri patrimonii in dicto regno Maioricarum cuius industriam, probitatem et fidem a mag. 
no citra tempore et in diversis curie nostre officiis et administrationibus experti sumus. Vos igitur eundem Gasparem tenorem presentium ad maiora promoventes ex conservatore nostri patrimonii de quodquidem officio ob hanc promotionem alteri providendum duximus MAGISTRUM RACIONALEM curie nostre in regno Maioricarum de NOVO CONSTITUIMUS, CREAMUS et pariter ORDINAMUS ex certa nostra sciencia et consulto motuque proprio et ex potestatis nostre plenitudine legibus non summissa, cum eis omnibus et singulis preeminenciis, honoribus, prerogativis, potestatibus et jurisdictionibus et emolumentis ad dictum officium spectantibus et pertinentibus sic et prout ac eis modo et forma quibus Magister Racionalis curie nostre in regno Valencie officium ipsum Magistri Racionalis in illo regno possidet acque melius tenet cum velimus quod forma qua institutum est illud officium hoc in ipso regno instituatur cum annuo tamen salario et ministris aliis nostris litteris declarandis prestito tamen per vos in posse Gubernatoris ipsius regni ad sancta Dei Evangelia juramento ac solenniter ut moris est de bene scilicet fideliter et legaliter vos habendo in regimine et exercicio dicti officii ad honorem quidem et servicium nostrum ac nostre curie jurium utilitatem comodum vel augmentum. Ita quod vos dictus Gaspar et nemo alius vita vestra durante sitis Magister Racionalis in dicto regno Maioricarum et ea omnia faciatis que Magistri Racionales curie nostre faciunt ac facere possunt et debent ipsumque officium teneatis, regatis et exerceatis prout de vobis confidimus fideliter, legaliter acque bene ut prefertur cum omnibus et singulis honoribus preeminenciis, potestatibus, auctoritatibus, exerciciis, superioritatibus, jurisdictionibus, libertatibus, immunitatibus et omnibus cunctisque aliis eidem officio debitis, consuetis et pertinentibus et cum quibus ac prout dictus Magister Racionalis regni Valencie illud officium in illo regno possidet utilitatem semper nostram et curie nostre procurando.

Illustrissime propterea regine consorti nostre maiestatis et locum tenenti generali nostre intentum nostrum super his declaramus quo volumus, disponimus et jubemus Gubernatori vero et aliis officialibus nostris in dicto regno constitutis dicimus, precipimus et stricte precipiendo, mandamus harum serie et ex certa nostra sciencia et consulte quatenus visis presentibus nulloque a nobis alio spectato mandato vos eundem Gasparem in possessionem dicti officii ponant et immittant positumque et immissum manuteneant et defendant quibusvis in contrarium allegandis non obstantibus ullomodo quibus de certa sciencia et consulte derogamus per presentes. Insuper earundem tenore presentium et ex certa nostra sciencia et consulto, mandamus procuratori regio dicti regni Maioricarum presenti et successive futuris eorumque locatenentibus et aliis universis et singulis administratoribus rerum nostrarum seu curie nostre pertinentium in dicto regno quatenus ex hinc 
in antea vobis dicto Magistro Racionali et nemini alteri compota eorum administrationum reddant vosque pro Magistro Racionali curie nostre in dicto regno habeant reputent acque tractent vobisque pareant et obediant in cunctis officio vestro pertinentibus et spectantibus. Contrariumque minime facturi quanto dicta serenissima consors nostra servicium nostrum carum habet et gubernator ac alii officiales et administratores predicti graciam nostram caram habent et penam confiscacionis omnium bonorum que possident et amissionis officiorum quibus fruuntur cupiunt evitare. In cuius rei testimonium presentes fieri jussimus acque ordinavimus nostro comuni sigillo impendenti munitas. Datum in Castello Novo civitatis Neapolis die decimo octavo mensis januarii millesimo $\mathrm{CCCC}^{\circ}$ quinquagesimo primo, regni huius citra farum Sicilie anno $\mathrm{XVII}{ }^{\circ}$ aliorum vero regnorum nostrorum tricesimo sexto. Rex Alfonsus.

Verum cum in preinserto concessionis et commissionis privilegio sit expresse declaratum quod Magister Racionalis curie nostre in dicto regno utatur acque uti debeat possitque illis preeminenciis, honoribus, prerogativis, potestatibus et jurisdictionibus acque habeat et recipiat ea emolumenta et jura ad dictum officium spectantia et pertinencia ac sic et prout acque eis modo institutione et forma quibus Magister Racionalis curie nostre in dicto regno Valencie officium ipsum tenet, possidet acque exercet cum annuo salario tamen dicti Magistri Racionalis et eiusdem officii ministrorum aliis nostris litteris declarando ut in dicto preinserto privilegio lacius hec omnia expressata sunt. Volentes ic circo id quod in dicto privilegio declarandum promissimus cum presenti declarare, considerantes presertim causas que ad hoc officium in dicto regno statuendum nos induxerunt et utilitatem curie nostre ea de causa futuram satis aperte videntes:

Tenore presentis nostri privilegii cunctis temporibus firmiter valitu$r i$ ex certa sciencia et consulto ac motu proprio privilegium preinsertum et omnia in eo contenta confirmantes et de novo concedentes statuimus, constituimus, declaramus et ordinamus quod in dicto Magistri Racionalis officio sint tamen ministri infrascripti et non plures. Scilicet, Magister Racionalis, duo scribe et unus virgarius, quorum scribarum alter sit locumtenens et obtineat primum locum in dicto officio immediate post dictum Magistrum; et absente Magistro ea omnia faciat et facere possit que ipse Magister Racionalis si adesset facere posset et quorum scribarum et virgarii nomina aliis nostris privilegiis cuique ipsorum de suo officio singulariter faciendis declaranda duximus; ipsi vero Magister, duo scribe et virgarius habeant pretextu et occasione sive racione officiorum predictorum salaria infrascripta videlicet Magister Racionalis habeat anno quolibet libras monete dicti regni trecentas; primus scriba et locumtenens, centum; secundus septuagintaquinque et virgarius sive portarius, vigintiquinque et non ultra. Liceat tamen 
dicto Magistro Racionali si opus fuerit plures ministros sive scribas in dicto officio ordinare extraordinarios tamen et sine salario; volumus insuper et expresse declaramus quod ipsi Magister Racionalis vel scribe teneantur et debeant audire, examinare, discutere acque videre, revidere, impugnare, notare, indiscussa dimittere et super illis consultare et alias vel expost concludere vel admittere ac deffinire in totum vel in partem omnia compota et raciones administrationum quarumcumque tam procuratoris regii dicti regni Maioricarum et insularum ei adiacentium veluti Minoricarum et Ebusii perinde ac faciebat et facere debebat Magister Racionalis curie nostre in Principatu Cathalonie quam eciam aliorum omnium baiulorum, collectorum, exactorum, procuratorum vel aliorum administratorum et officialium tam ad annum sive ad beneplacitum vel ad vitam per nos, nostros locumtenentes generales seu alios inde potestatem habentes ordinatorum et ordinandorum in dicto regno Maioricarum officiis quorum debeatur seu quavis racione incumbat administracio seu receptio qualiscunque pecuniarum et jurium predictorum in dicto regno et insulis constiturom presentium et futurorum sic et prout faciebat et facere debebat procurator regius dicti regni per quem hactenus raciones ipsorum particularium administratorum in dicto regno audiebantur et diffinebantur que compota et raciones predicte in dicto officio Magistri Racionalis ut predicitur audiende, examinande et admittende vel repellende ac diffiniende audiantur, discutentur, examinentur, admittantur ac diffiniantur vel repellantur intus castrum regium civitatis Maioricarum in quo volumus ipsum officium Magistri Racionalis locum proprium dicatum, aptum et sufficientem ad id specialiter deputatum habere acque tenere in quo loco sit Archivus, ubi libri, quaterni, cautele, apoce et scripture alie quevis que in dicto officio fiunt seu dantur et restituuntur comode, tute ac secreto reponantur custodianturque qui locus reperiatur acque ordinetur statim acque omni postposita mora in dicto castro in ea eiusdem castri parte que magis conveniens et oportuna reperiatur et constituatur ad arbitrium Gubernatoris sive eiusmodi officium regentis vel locumtenentis et dicti Magistri Racionalis, procuratoris quoque regii et conservatoris nostri patrimonii in dicto regno. Volumus propterea ac demum declaramus ex eadem certa sciencia nostra quod ipse Magister Racionalis in dicto regno Maioricarum et ipsius officii et ministri et alii ad quos pertineat debeant atque teneantur servare ordinationes, statuta et institutiones omnes et quascunque quibus officium Magister Racionalis curie nostre in dicta nostra curia statutum acque ordinatum est ac eis omnibus quibus alii Magistri Racionales qui hactenus generaliter et particulariter ordinati fuerunt melius servarunt et servare debuerunt. Et pari forma ipsi Magister et ministri utantur gaudeantque omnibus illis superioritatibus, prerogativis, honoribus, preeminenciis et favoribus quibus ab antiquo habentes tale offi- 
cium sive Magistratum usi fuerunt, utuntur acque melius uti debent et possunt omni juris vel facti contradictione cessante. Cum sic ex mera nostra voluntate acque consilio procedat. Illustrissime propterea Regine consorti et locumtenenti generali nostre in regnis et terri nostris occiduins aliisque insuper locumtenentibus nostris generalibus per has easdem intentum et voluntatem nostram aperimus, Gubernatori quoque dicti regni Maioricarum seu eius locumtenenti vel ipsum offcium regenti, Procuratori eciam regio et conservatori nostri patrimonii et advocato et procuratori fisci nostri, baiulo insuper et vicario ac juratis et administratoribus, exactoribus et perceptoribus pecuniarum et rerum quarumcunque nostre curie quoquomodo spectantium aliisque eciam universis et singulis officialibus et subditis nostris in dicto regno constitutis presentibus et futuris mandamus quatenus presentem nostram declarationem, institutionem et ordinationem omnia denique desuper contenta prout jacent omni sinistra interpretatione remota servent et servari faciant per quoscunque. Et dictus procurator regius sive eius locumtenens vel ipsum officium regens qui nunc est et pro tempore fuerit de salario sive salariis desuper dicto Magistro Racionali et ministris constituto sive constitutis a die date privilegii preinserti in presentem usque diem respondeat respondereque teneatur et debeat cavillacione quacunque reierca et hinc in antea de tercia in terciam salaria ipsa dicto Magistro Racionali et ministris solvat integre et absque alicuius diminucione. Recuperaturus ab eisdem Magistro Racionali et ministris debitas apodixas in quarum prima tenor huiusmodi inseratur et in aliis tamen fiat mencio de eadem specialis in ipsius procuratoris regii comportis admittendas. Aliaque omnia ipse procurator faciat que racione dicti sui officii erga dictum Magistrum Racionalem tam in exhibendis comportis quam alias facere debet et tenetur hocque neque ic ipse neque alii predicti muttent quavis causa. In quorum testimonium presentes fieri jussimus comuni nostro pendenti sigillo munitas. Datum in Turri Octave die vicesimo quinto mensis octobris anno a Nativitate Domini $\mathrm{M}^{\circ} \mathrm{CCCC}^{\circ}$ quinquagesimo primo regnique huius Sicile citra Farum anno $\mathrm{XVII}^{\circ}$ aliorum vero regnorum nostrorum tricesimo sexto. Rex Alfonsus.

Dominus rex mandavit mihi Arnaldo Fonolleda et viderunt Petrus de Capdevila Thesaurarius et Petrus, regii patrimonii generalis conservator. Probata'.

I ACA, Reg. Cancillería núm. 2918, fol. 28 v. 
Executoria precedentis privilegii.

Alfonsus etc. Illustrissime Regine Marie consorti carissime et locumtenenti nostre generali in regnis et terris nostris occiduis prosperos ad vota successus aliisque insuper locumtenentibus nostris generalibus, gubernatori quoque dicti regni Maioricarum seu eius locumtenenti vel ipsum officium regenti, procuratori etiam regio et conservatori nostri patrimoni et advocato ac procuratori fisci nostri, baiulo, vicario et juratis ac administratoribus, exactoribus et perceptoribus pecuniarum et rerum quarumcunque curie nostre quoquomodo spectantium et pertinentium alisque universis et singulis officialibus et subditis nostris in dicto regno Maioricarum constitutis presentibus et futuris eorumque locatenentibus. Salutem et dilectionem. Ecce quod Nos die presenti et infrascripto confirmavimus, instituimus et iterum ac de novo ordinaviums Magistrum Racionalem curie nostre in isto regno Maioricarum dilectum Consiliarium nostrum GASPAREM DE PACHS, cum salario ministris et ministrorum salariis ac aliis in dicto confirmationis privilegio die presenti ut prefertur dato contentis et expressatis, sitque nostre firmissime intentionis quod dictum privilegium et omnia in eo contenta suum sorciantur effectum ac per vos et vestrum quemlibet teneantur et ad unguem observentur, vobis dicte serenissime consorti et locumtenentis generali nostre intentum nostrum super predictis declaramus quo volumus, disponimus et jubemus aliis vero predictis et presertim vobis dicto procuratori regio vel vestro locumtenenti dicimus et stricte precipiendo mandamus ad penam florenorum decem mille a bonis contrafacientis cuiuslibet irremissibiliter exigendorum et nostre curie applicandorum privationemque officiorum per nos vobis commissorum quas ilico contravencionis casu incurratis in eisque incidatis quatenus predictum confirmacionis et de novo concessionis privilegium juxta sui seriem et tenorem et omnia in eo contenta dicto Gaspari de Pachs Magistro Racionali qui supra concessum teneatis, exequamini et adimpleatis tenerique, exequi et adimpleri per quos deceat faciatis. Et contrarium non faciatis si vos dicta Serenissima consors et locumtenens nostra generalis nobis complacere et servire vos vero alii predicti graciam nostram caram habens iramque et indignacionem ac penam et privationem desuper contentas quibus pronta et rigida non deerit executio cupitis non incurrere. Cum sic ex mente nostra procedat omnibus dubio contradictione et consultatione cessantibus et reiectis. Datum in Turri Octave die $\mathrm{XXV}^{\circ}$ mensis octobris anno a Nativitate Domini $\mathrm{M}^{\circ} \mathrm{CCCC}^{\circ} \mathbf{L l}^{\circ}$ : Rex Alfonsus.

Dominus rex mandavit mihi Arnaldo Fonolleda et vidit Petrus, regii patrimonii generalis conservator. Probata ${ }^{1}$.

' ACA, Reg. Cancilleria núm. 2981, fol. 31 v. 
Nos don Alfonso etc. Universes et sengles officials e subdits nostres en la ciutat e regne de Malorques constituhits presents e sdevenidors. Saluts e dilecció. Havents a memoria quants dans se son seguits en lo regne de Mallorques per les contencions que sobre lo regiment de aquell regne entre los ciutadans incoles e habitadors de la dita ciutat e regne son stades e volents provehir quant en nos sia que no haien occasió de mes contendre havents de nou creat en lo dit regne per utilitat de nostra cort OFFICI de MESTRE RACIONAL e aquell comes al amat conseller nostre en GASPAR de PACHS lo qual offici sens dupte algu es superior al offici de Procurador reyal del dit regne e lo qui es Mestre Racional deu precehir al dit Procurador real en totes honors e prerogatives, emperho considerants que l-offici de Procurador real es antiquissim en aquell regne e nos quant lo atorgam al amat conseller e maiordom nostre mossen Johan Albertí e lo y donam que no.y havia Mestre Racional algú que 1 precehis; e com ara de nou lo haiam fet quasi seria vist esser estat fet en preiudici e derogació sua e de son offici, havem delliberat e axi los dits Mestre Racional e Procurador real qui son ensems presents en nostra cort, son contents de la present declaració, ço es, que vivint lo dit mossen Johan Albertí e tenint 1 -offici de Procurador real e exercici de aquell, no sia fet carech en la precedencia, ans preceescha al dit Mestre Racional en totes coses e lo dit Mestre Racional vaia, sega e succesca inmediadament apres lo dit Procurador real e apres del dit Mestre Racional inmediadament lo Conservador de nostre patrimoni en lo dit regne venint enpero quanquequant primer lo dit offici de Procurador real vendra en altres mans per mort, renunciació, resignació, suspenció o altra qualsevol causa per la present no entenem preiudicar als dits officis de Mestre Racional e Conservador, als quals de present per servar una equitat la qual es procehida de voluntat dels dits Mestre Racional e Procurador Real havem derogat, ans volem e ab la present ordenam e declaram de nostre bon grat e de certa sciencia, que lo Mestre Racional preceesca al Procurador Real e lo Procurador real al Conservador segons vol la rahó e es estat per nos ordenat en la creació dels officis de Mestre Racional e de Conservador. E aquesta present declaració e ordinació volem segons dessus es ordenat sia en lo dit regne servada, remoguda tota contradicció e així manant ab les presents de nostre bon grat e certa sciencia als dits Mestre Racional, Procurador Real e Conservador e altres officials e persones de qui sia enterès e pena de deu milia florins que ho serven quietament per tot lo temps que mossen Albertí será personalment en lo dit regne e exercici de son offici; declarat empero que si lo dit mossen Albertí será absent e en son loch vendrá algún regent o loctinent, lo dit offici de Procurador Real aquell tal no volem preceesca al dit Mestre Racional, ans sia precehit per lo dit Mestre 
Racional. Manant a vosaltres e a cascú de vosaltres sots les dites penes que la present ordinació, declaració e graduació, com dit es, de concordia dels dits Mestre Racional e Procurador Real feta, serven e servar facen a la letra com aquesta sia nostra volentat. Dada en la Torre d.Octavo a XXV del mes de octubre del any de la Nativitat de Nostre Senyor M CCCC LI. Rex Alfonsus.

Dominus rex mandavit michi Arnaldo Fonolleda. Probata ${ }^{1}$.

1 ACA, Reg. Cancilleria núm. 2918, fol. 40 v. 